\title{
Room-temperature electroluminescence from electron-hole plasmas in the metal-oxide-silicon tunneling diodes
}

\author{
C. W. Liu, ${ }^{\text {a) }}$ M. H. Lee, Miin-Jang Chen, ${ }^{\text {b) }}$ I. C. Lin, and Ching-Fuh Lin ${ }^{\text {b) }}$ \\ Department of Electrical Engineering, National Taiwan University, Taipei, Taiwan, Republic of China
}

(Received 30 August 1999; accepted for publication 28 January 2000)

\begin{abstract}
An electron-hole plasma recombination model is used to fit the room-temperature electroluminescence from metal-oxide-silicon tunneling diodes. The relatively narrow line shape in the emission spectra can be understood by the quasi-Fermi level positions of electrons and holes, which both lie in the band gap. This model also gives a narrower band gap than that of bulk silicon. The surface band bending in the Si/oxide interface is responsible for this energy gap reduction. (C) 2000 American Institute of Physics. [S0003-6951(00)04512-5]
\end{abstract}

It is a long sought goal to integrate ultralarge scale integrated (ULSI) circuits with the electro-optics to possibly overcome the speed limitation of electrical interconnects and reduce the complexity of the backend process. Although strained silicon germanium technology has been reported to serve this purpose, ${ }^{1}$ the fabrication complexity and cost of this technology make it difficult to implement in a real chip. Recently, the observation of light emission from the metaloxide-silicon (MOS) tunneling diodes ${ }^{2,3}$ sheds new light on this goal. However, to further utilize this electroluminescence (EL), it is essential to understand the physical mechanism during the light emission process. In photoluminescence (PL), it has been reported that electron-hole plasma recombination is the dominating mechanism in some indirect semiconductors $^{4-6}$ such as $\mathrm{SiGe}, \mathrm{AlGaAs}, \mathrm{SiC}$, and GaP. For example, Xiao et al. got an excellent fit of the PL spectra from the $\mathrm{Si} / \mathrm{SiGe} / \mathrm{Si}$ quantum wells using an electron-hole plasma (EHP) model at $77 \mathrm{~K}$. Previous studies of EL from $\mathrm{SiGe/Si} p$-type-intrinsic- $n$-type $(p-i-n)$ diodes by Mi et al. ${ }^{1}$ and Robbins et al., ${ }^{7}$ a no-phonon (NP) emission as well as a transverse phonon (TO) replica is observed. However, the $p-i-n$ structure is not fully compatible with ULSI process. The building device in ULSI is the MOS structure. We, therefore, investigate the line shape of the room-temperature EL from the MOS tunneling diodes for $n$-type $\mathrm{Si}$ as well as $p$-type Si. The MOS tunneling diode has exact the same structure used in the ULSI circuits, while the oxide is thin enough to have significant tunneling current from the metal to the silicon substrate.

The ultrathin gate oxide used in this study is grown by rapid thermal oxidation at the $900^{\circ} \mathrm{C}$. The gas flows are 500 sccm nitrogen and $500 \mathrm{sccm}$ oxygen at a reduced pressure. The thickness is measured by ellipsometry, confirmed by high frequency capacitance-voltage measurements. The resistivity of the $100 \mathrm{~mm} n$-type wafers is $1-10 \Omega \mathrm{cm}$. The p-type MOS (PMOS) diodes had Al gate electrodes with various circular areas defined by photolithography.

Figure 1 shows the typical current-voltage $(I-V)$ curves of a PMOS diode with the oxide thickness of $2.7 \mathrm{~nm}$. When the $\mathrm{Al}$ gate with the area of $3 \times 10^{-4} \mathrm{~cm}^{2}$ was under

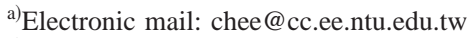

${ }^{b)}$ Also with: Institute of Electro-Optical Engineering.
}

positive bias, the electrons tunneled from the $n$-type silicon to the $\mathrm{Al}$ gate and the holes also tunneled from $\mathrm{Al}$ gate to $n$-type silicon. As a result, a significant tunneling current was observed in the positive gate bias. However, due to the different barrier heights between electrons $(\sim 3.1 \mathrm{eV})$ and holes $(\sim 5.6 \mathrm{eV})$, hole current may be smaller than the electron current. Meanwhile, the positive gate bias also attracts electrons in the silicon/oxide interface to form an accumulation layer. The localized electrons in the accumulation layer have localized wave functions, which can have a spread in the reciprocal space and may provide extra momentum during the electron-hole radiative recombination. When the Al gate is under negative gate bias, the current is controlled by the thermal generated holes and is independent of negative gate bias. $^{2}$

When significant current flowed through the PMOS structure under positive gate bias, luminescence was clearly observed under continuous wave operation without cooling. Since the light was blocked by the thick Al gate electrode, only the emission from the diode edge was detected, as observed by infrared viewer through a microscope. Figure 2 shows the emission spectra of an $\mathrm{Al} / 2.7 \mathrm{~nm}$ oxide $/ n$-silicon PMOS diode with a circular area of $4 \times 10^{-2} \mathrm{~cm}^{2}$. The emission intensity increases with the positive gate bias. The peak width of our spectra increases from 68 to $86 \mathrm{meV}$, as the gate

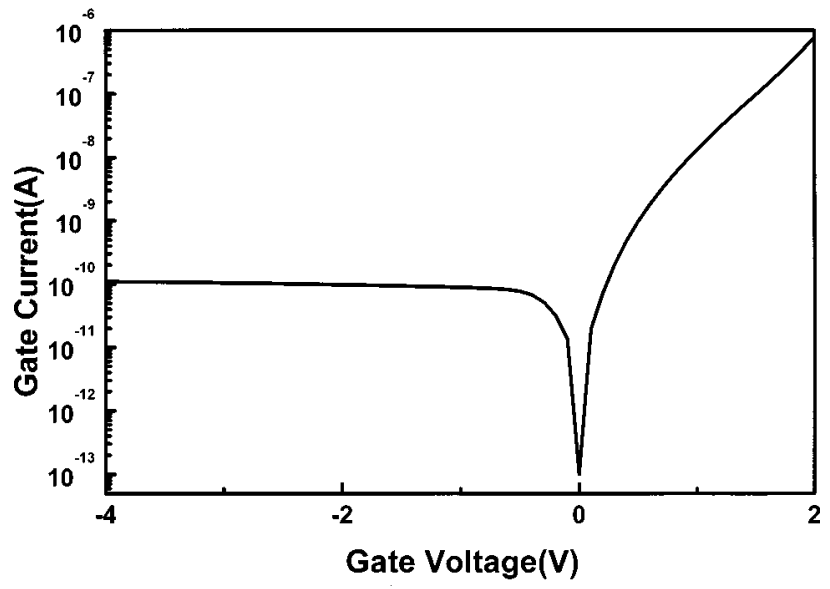

FIG. 1. The I-V curve of a PMOS diode. The EL is observed under positive gate bias. 


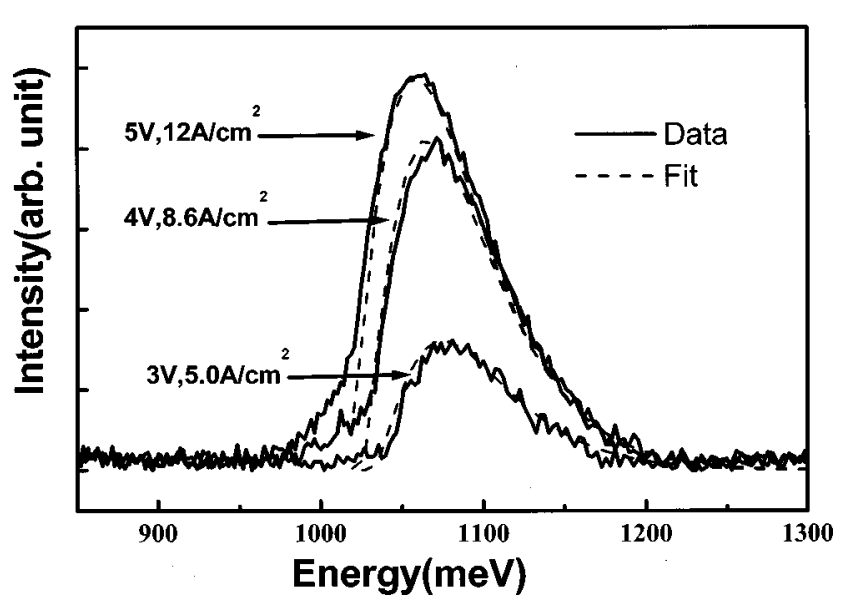

FIG. 2. The theoretical fitting of EL spectra from a PMOS tunneling diode.

bias increase from 3 to $5 \mathrm{~V}$. This width is about half of the previous data of the room-temperature spectra from the $\mathrm{Si} /$ SiGe $p-i-n$ diode. ${ }^{1}$ The feature of the emission spectra is the asymmetrical broadening of the emission peak, where the high-energy part is broader than the low-energy part. This is very similar to the line shape of electron-hole-plasma recombination. ${ }^{4,5}$ To confirm this observation, we fit the EL spectra by the line shape of EHP recombination using the following expression:

$$
\begin{aligned}
I(h \nu)= & I_{0} \int_{0}^{h \nu-E_{g, \mathrm{EL}}} d E D_{e}(E) D_{h}\left(h \nu-E_{g, \mathrm{EL}}\right. \\
& -E) f\left(E, F_{e}, T\right) f\left(h \nu-E_{g, \mathrm{EL}}-E, F_{h}, T\right),
\end{aligned}
$$

where $D_{e}$ and $D_{h}$ are the densities of states of electron and hole, respectively, $F_{e}$ and $F_{h}$ are the respective quasi-Fermi energy, $h \nu$ is the energy of photon emitted, $T$ is the measurement temperature, $E_{g, \mathrm{EL}}$ is the band gap of Si obtained by the EL measurements, and the $f$ 's are the Fermi-Dirac distribution. In this expression, only the conservation of energy is taken into account. The momentum conservation required by the indirect bandgap nature of $\mathrm{Si}$ is assumed to be provided by the scattering of Si/oxide roughness ${ }^{8}$ and/or the spread in the reciprocal space ( $k$ space) due to the localized wave function of electrons in the accumulation layer. The threedimensional density of states $\left[D(E) \sim E^{1 / 2}\right]$ is used for holes, while a constant two-dimensional density of states is used for electrons in the accumulation layer. There are four adjustable parameters for the fitting: the relative intensity $I_{o}$, the band gap $E_{g, \text { EL}}$, and the quasi-Fermi energy $F_{e}$ and $F_{h}$. The band gap determines the energy position of the spectra, and quasiFermi energies determine the line shapes of the spectra. A narrow peak is obtained, if the quasi-Fermi levels of electrons and holes lie closely in the band gap. A wide peak is obtained, if the quasi-Fermi levels of electrons and holes move deeply into the conduction band and the valence band, respectively. Figure 2 also shows the theoretical EHP spectra, indicating an excellent fit to the experimental data. The fitting parameters obtained in this work are quite different from those of the previous report. ${ }^{4,5}$ The quasi-Fermi energies for both electrons and holes in our simulation lie within the band gap, while the quasi-Fermi energies of electrons and holes move into conduction band and valence band, re-

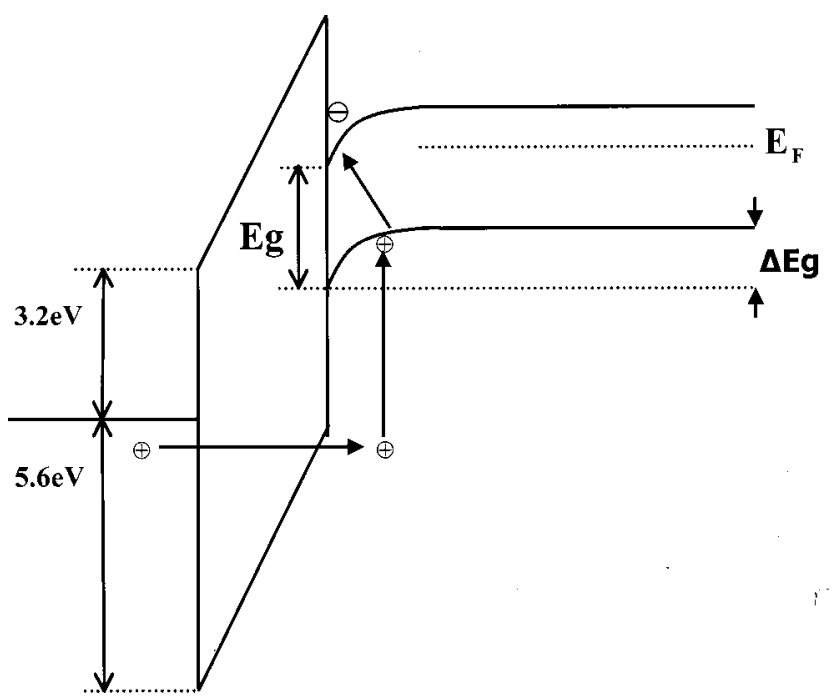

FIG. 3. The schematic band diagram in the PMOS diode to explain the band gap reduction, obtained from the EHP model.

spectively, in the previous work. ${ }^{4,5}$ This indicates that the electron and hole concentrations in the tunneling PMOS diodes are much smaller as compared to the $\mathrm{Si} / \mathrm{SiGe} / \mathrm{Si}$ quantum wells. As a result, the peak width in our work is much smaller, as compared to the previous reported data. The band gap obtained from the EHP model is about $1.01-1.03 \mathrm{eV}$ for the gate voltage from 5 to $3 \mathrm{~V}$, lower than the Si band gap of $1.12 \mathrm{eV}$ at room temperature. This is unlikely to due to high junction temperature in Si/oxide interface, since the temperature of $1.01 \mathrm{eV}$ band gap is about $600 \mathrm{~K}$ and the EL spectra can not be fitted well using such high temperature. This reduction of band gap can be understood by the surface band bending in the PMOS band diagram under the accumulation condition as shown in Fig. 3. The tunneling holes from the $\mathrm{Al}$ gate electrode injected into the valence band of $n$-Si will not stop at the interface, can probably reach the neutral region of $n-\mathrm{Si}$ and relaxed to the valence band edge in the neutral region. The recombination of holes in the neutral region with the electrons in the accumulation region can emit photons with energy of the difference between the Si band gap and the surface band bending.

Besides the main emission peak (NP), the relatively

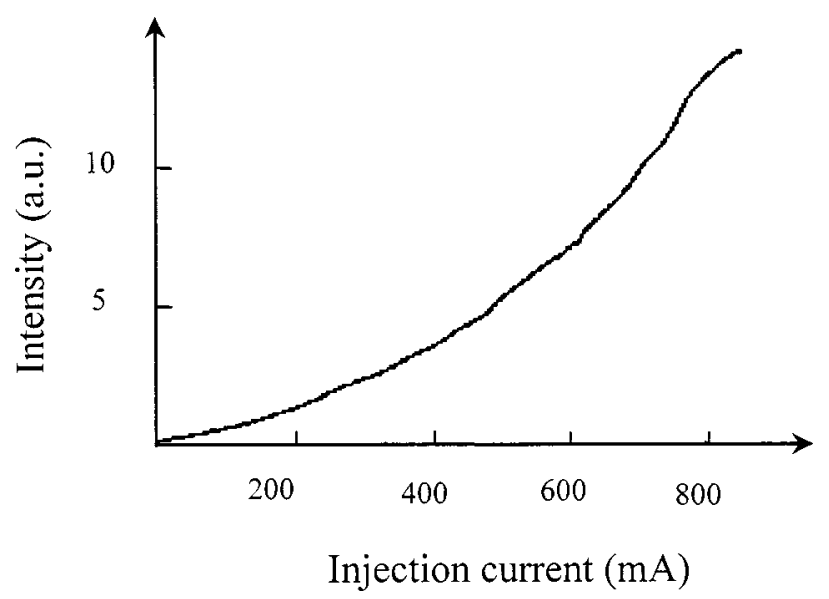

FIG. 4. The dependence of peak intensity on current drive for a PMOS tunneling diode. 


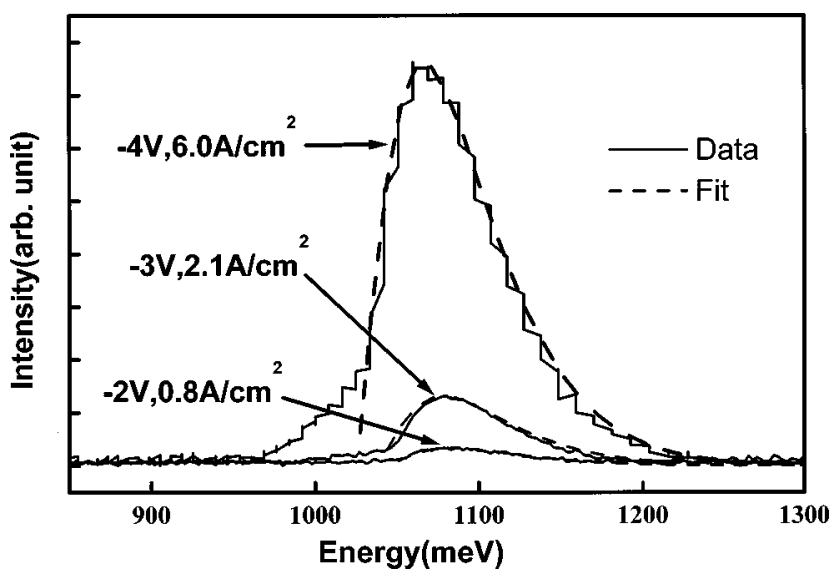

FIG. 5. The theoretical fitting of EL spectra from a NMOS tunneling diode.

week TO replica was also observed in PL spectra of the bulk of $\mathrm{Si}^{4}{ }^{4}$ relaxed $\mathrm{SiGe},{ }^{9}$ and strained $\mathrm{SiGe} .{ }^{4,5}$ However, only one peak is observed in the EL spectra of the PMOS diode. The origin is not clear.

The dependence of the peak EL intensity on drive current is shown in Fig. 4. There is a superlinear increase of the output power with injection current. The efficiency seems to be enhanced at high current level. However, it is difficult to estimate the quantum efficiency, since the $\mathrm{Al}$ gate electrode blocks most light, and only the light emitted from the diode edge can be detected.

The EHP model also gives an excellent fit to EL spectra $^{2,3}$ of the $n$-type MOS (NMOS) tunneling diode as shown in Fig. 5. The band gap obtained from the fitting is about $1.02-1.04 \mathrm{eV}$ for the negative gate bias from 5 to 2.5 $\mathrm{V}$, lower than the Si band gap. Similarly, the surface band bending is also responsible for this reduction

In summary, we presented the EL from the electron-hole plasma recombination in the MOS tunneling diodes. The EL spectra can be well fitted by a simple convolution of electron and hole distribution functions. The surface band bending in the Si/oxide interface is responsible for the reduced band gap, obtained from the EHP model. The relative narrow line width can be also understood by the fact that the positions of the quasi-Fermi energies of electrons and holes are both in the band gap.

This work is supported in part by National Science Council, Taiwan, Republic of China (Nos. 88-2218-E-002004 and 2112-M-002-038). The support of UMC and TSMC is also highly appreciated.

${ }^{1}$ Q. Mi, X. Xiao, J. C. Sturm, L. C. Lenchyshyn, and M. L. W. Thewalt, Appl. Phys. Lett. 60, 3177 (1992).

${ }^{2}$ C. W. Liu, M. H. Lee, C. F. Lin, I. C. Lin, W. T. Liu, and H. H. Lin, Tech. Dig. Int. Electron Device Meet. 749 (1999).

${ }^{3}$ C.-F. Lin, C. W. Liu, M.-J. Chen, M. H. Lee, and I. C. Lin, J. Phys.: Condens. Matter (in press).

${ }^{4}$ X. Xiao, C. W. Liu, J. C. Sturm, L. C. Lenchyshyn, and M. L. Thewalt, Appl. Phys. Lett. 60, 1720 (1992).

${ }^{5}$ C. W. Liu, J. C. Sturm, Y. R. J. Lacroix, M. L. W. Thewalt, and D. D. Perovic, Appl. Phys. Lett. 65, 76 (1994).

${ }^{6}$ E. Cohen, M. D. Sturge, M. A. Olmstead, and R. A. Logan, Phys. Rev. B 22, 771 (1980).

${ }^{7}$ D. J. Robbins, P. Calcott, and W. Y. Leong, Appl. Phys. Lett. 59, 1350 (1991).

${ }^{8}$ M. Jaros and A. W. Beavis, Appl. Phys. Lett. 63, 669 (1993).

${ }^{9}$ J. Weber and M. I. Alonso, Phys. Rev. B 40, 5683 (1989). 